\title{
Association between the Angiotensin-Converting Enzyme (ACE) Genetic Polymorphism and Diabetic Retinopathy-A Meta-Analysis Comprising 10,168 Subjects
}

\author{
Shasha Luo ${ }^{1,+}$, Chao Shi ${ }^{2,+}$, Furu Wang ${ }^{3, *}$ and Zhifeng $\mathrm{Wu}^{1, *}$ \\ 1 Department of Ophthalmology, Nanjing Medical University Affiliated Wuxi Second Hospital, \\ 68 Zhongshan Road, Wuxi 214002, China; luoshasha403@163.com \\ 2 Wuxi Center for Disease Control and Prevention, 499 Jincheng Road, Wuxi 214023, China; \\ shichao.xo@163.com \\ 3 Jiangsu Provincial Center for Disease Prevention and Control, 172 Jiangsu Road, Nanjing 210029, China \\ * Correspondence: furuwang83@163.com (F.W.); zhifengwu2013@126.com (Z.W.); \\ Tel./Fax: +86-510-6668-177 (Z.W.) \\ + These authors contributed equally to this work.
}

Academic Editor: William Chi-shing Cho

Received: 11 August 2016; Accepted: 2 November 2016; Published: 15 November 2016

\begin{abstract}
Aims-to address the inconclusive findings of the association of angiotensin-converting enzyme (ACE) insertion/deletion (I/D) polymorphism on risk of diabetic retinopathy (DR), a meta-analysis was conducted. Methods-we conducted a meta-analysis on 4252 DR cases and 5916 controls from 40 published studies by searching electronic databases and reference lists of relevant articles. A random-effects or fixed-effects model was used to estimate the overall and stratification effect sizes on ACE I/D polymorphism on the risk of DR. Results-we found a significant association between the ACE I/D polymorphism and the risk of DR for all genetic model (ID vs. II: OR $=1.14$, 95\% CI: 1.00-1.30; DD vs. II: OR = 1.38, 95\% CI: 1.11-1.71; Allele contrast: OR $=1.17,95 \%$ CI: 1.05-1.30; recessive model: $\mathrm{OR}=1.24,95 \%$ CI: $1.02-1.51$ and dominant model: $\mathrm{OR}=1.21,95 \%$ CI: $1.06-1.38$, respectively). In stratified analysis by ethnicity and DM type, we further found that the Asian group with T2DM showed a significant association for all genetic models (ID vs. II: OR $=1.14,95 \%$ CI: 1.01-1.30; DD vs. II: OR $=1.54,95 \%$ CI: $1.14-2.08$; Allele contrast: $\mathrm{OR}=1.26,95 \%$ CI: $1.09-1.47$; recessive model: $\mathrm{OR}=1.42,95 \% \mathrm{CI}: 1.07-1.88$ and dominant model: $\mathrm{OR}=1.26,95 \%$ CI: $1.07-1.49$, respectively). Conclusion-our study suggested that the ACE I/D polymorphism may contribute to DR development, especially in the Asian group with type 2 diabetes mellitus (T2DM). Prospective and more genome-wide association studies (GWAS) are needed to clarify the real role of the ACE gene in determining susceptibility to DR.
\end{abstract}

Keywords: ACE I/D; polymorphism; diabetic retinopathy; DM type; ethnicity

\section{Introduction}

Diabetic retinopathy(DR) is the premier cause of vision loss in adults aged 20-74 years [1]. From 1990 to 2010, DR ranked as the fifth most accpeted cause of preventable blindness and moderate to severe visual impairment [2]. Diabetic retinopathy (DR) is a microvascular complication occurring both in type 1 diabetes mellitus (T1DM) and type 2 diabetes mellitus (T2DM), and it was estimated that, of 285 million people worldwide with diabetes, over one-third had signs of DR in 2010 [3]. DR is a complex trait involving polygenic, metabolic, and environmental influences. Known risk factors, most notably the duration of diabetes and glycemic control, explain some, but not all, of the progression 
of DR [4-6]. There are diabetic patients with DR despite short durations of diabetes and/or perfect glycemic control and other diabetic patients who do not develop DR in the face of long-standing diabetes and/or long-term hyperglycemia [7]. Therefore, the genetic factor may explain some of the variation in the progression of DR [8].

The angiotensin-converting enzyme (ACE) gene, plays an critical role in modulating vascular tone through hydrolyzing angiotensin I to vasoconstrictory peptide angiotensin II, which seems to be particularly biologically and clinically relevant to diabetes [9]. A number of studies have reported that patients suffering from DR have high circulating levels of ACE, which implies that elevated serum ACE levels might be a possible hazard factor in destroying retinal vascular apparatus in subjects suffering from diabetes [10]. The ACE gene has a frequent insertion/deletion (I/D) polymorphism characterized by the presence or absence of a $287 \mathrm{bp}$ Alu repetitive sequence in intron 16 [11]. This polymorphism was associated with circulating ACE levels and increased plasma and tissue activity of this enzyme [11-13]. Because of the central role of the ACE gene, it is feasible to hypothesize that polymorphism of ACE I/D contributes to the development of DR and numerous studies have addressed the role of the variation in the complex etiology of DR.

Numbers of molecular epidemiological studies have been performed to examine the relationship between the ACE I/D polymorphism and DR [14-55], but the results remain inconclusive. Although several meta-analyses have been published [56,57], they still did not reach a consistent conclusion. To better shed light on these conflicting findings and to quantify the potential between-study heterogeneity and provide better ability to detect smaller effect sizes, we conducted a comprehensive meta-analysis on 40 published studies from 1994 to 2016 with 4252 diabetic retinopathy cases and 5916 controls relating the variant of the ACE I/D polymorphism to the risk of developing DR.

\section{Methods}

This study was reported according to the Meta-analysis of Observational Studies in Epidemiology (MOOSE) guidelines and Preferred Reporting Items for Systematic Reviews and Meta-Analyses (PRISMA) for reporting systematic reviews and meta-analyses. Study selection, data extraction, and quality assessment were completed independently by two investigators. Disagreement was resolved through discussion. If the discussion did not lead to a consensus, Professor Wu made the final decision.

\subsection{Identification and Eligibility of Relevant Studies}

All studies that determined the genotype distribution of ACE I/D polymorphism in cases with diabetes retinopathy, and (i) in diseased controls (subjects with diabetes and free of DR) or (ii) in healthy controls, were attempted to be included in the meta-analysis. Cases were type 1 or 2 diabetic subjects with background, simple, advanced, or proliferative DR. The control group consisted of two subgroups, the first was the diseased control group, which consisted of subjects with diabetes and which were free of diabetic retinopathy disease, i.e., diabetes nephropathy and myocardial infarct, and the second group was the healthy controls, which was made up of subjects without any diseases.

Studies were firstly identified by searching the electronic literature PubMed for relevant reports in English and CNKI for papers in Chinese (from January 1994 to April 2016, using the search terms "angiotensin converting enzyme" or "ACE" or "rs1799752" in combination with "diabetic retinopathy" or "diabetic retinopathies" or "DR"). We chose articles which were conducted among human subjects. Eligible studies were then identified by further searching the studies published to date on the association between $A C E \mathrm{I} / \mathrm{D}$ polymorphism and diabetic retinopathy risk, and restricted attention to the studies that satisfied all of the following criteria: studies related to the ACE polymorphism were determined regardless of sample size and study design (case-control, cross-sectional, or cohort studies); each genotype frequency was reported, and there was sufficient information for extraction of data; if studies had partly overlapped subjects, only the one with a larger and/or the latest sample size was selected for the analysis. Additional studies were identified by hands-on searches from references of original studies or review articles on this topic. According to these criteria, we finally included 40 papers in our meta-analysis. 


\subsection{Data Extraction and Conversion}

Two investigators independently extracted data and reached a consensus on all of the items. Data extracted from these articles included the first author's name, year of publication, study design, ethnicity of the study population, type of DM, clinical characteristics, and the number of cases and controls for ACE I/D genotypes. The frequencies of the alleles and the genotypic distributions were extracted or calculated for both cases and controls. We defined that diabetic patients without retinopathy and/or matched healthy persons constituted the control group, and patients with DR were the case group. We merged the original data into the control group or case group if the study did not provide corresponding data. For some studies without sufficient information for extraction of data, we tried to contact the studies' authors by sending emails to request data missing from their articles. In addition, it was tested whether the distribution of genotypes in the controls was consistent with the Hardy-Weinberg equilibrium (HWE) for each study, and calculated the frequency of the minor allele for ACE I/D polymorphism.

\subsection{Quality Assessment and Study Stratification}

The Newcastle-Ottawa scale (NOS) method was used to assess the observational included studies. The NOS is composed of three parts (8 entries): selection, comparability, and exposure. A quality item is given only one star for the study in selection and exposure, and a quality item is given, at most, two stars for the study in comparability. It is a semi-quantitative scale, and a score of 0-9 stars is assigned to each study. Studies whose scores were more than 6 stars were considered to be of relatively high quality [58]. The scores of included studies are shown in Table 1.

\subsection{Meta-Analysis}

The meta-analysis evaluated the relationship between the ACE I/D polymorphism and the risk of DR for each study by odds ratio (OR), with $95 \%$ confidence intervals $(95 \% \mathrm{CI})$. For all studies, we calculated the ORs for the: (i) separate pairwise comparisons; (ii) allele contrast; (iii) recessive model; and (iv) dominant model. In addition, we conducted stratification analysis by ethnicity and DM type. A sensitivity analysis, which examines the effect of excluding specific studies, was also performed [59]. Our meta-analysis was subjected to sensitivity analysis for studies with the controls not in HWE $(p<0.05)$.

The $\chi^{2}$-based $Q$ statistic test was used to assess the heterogeneity, and it was considered significant for $p<0.05$. Heterogeneity was quantified with the $I^{2}$ metric, which is independent of the number of studies in the meta-analysis. $I^{2}$ takes values between $0 \%$ and $100 \%$, with higher values denoting a greater degree of heterogeneity $\left(I^{2}>50 \%\right.$ was considered significant) [60]. We used the fixed-effects model and the random-effects model based on the Mantel-Haenszel method and the DerSimonian and Laird method, respectively, to combine values from each of the studies. When the effects were assumed to be homogenous, the fixed-effects model was then used; otherwise, the random-effects model was more appropriate [61]. In addition, we further conducted meta-regression analyses to estimate the source of heterogeneity. Publication bias was assessed according to the Egger regression asymmetry test and the Begg adjusted rank correlation test [62,63]. All analysis was done by using the Stata software (v.12.1) (StataCorp LP, College Station, TX, USA). All the $p$ values were two-sided. 
Table 1. Characteristics of published studies included in the meta-analysis.

\begin{tabular}{|c|c|c|c|c|c|c|c|c|c|c|c|c|c|c|}
\hline \multirow{2}{*}{$\begin{array}{c}\text { Author } \\
\text { (Reference) }\end{array}$} & \multirow{2}{*}{ Year } & \multirow{2}{*}{ Country } & \multirow{2}{*}{ Design } & \multicolumn{4}{|c|}{ Case } & \multicolumn{4}{|c|}{ Control } & \multirow[b]{2}{*}{ HWE\# } & \multirow{2}{*}{ MAF * } & \multirow{2}{*}{$\begin{array}{l}\text { NOS } \\
\text { (Stars *) }\end{array}$} \\
\hline & & & & Sample Size & Age (Year) & DM Duration (Year) & Definition & Sample Size & Age (Year) & DM Duration (Year) & Definition & & & \\
\hline $\begin{array}{c}\text { Marre } \\
\text { et al. [14]. }\end{array}$ & 1994 & France & $\mathrm{CC}$ & 52 & $39.0 \pm 14.0$ & $20.0 \pm 11.0$ & PDR & 32 & $43.0 \pm 18.0$ & $22.0 \pm 12.0$ & IDDM & 0.38 & 0.64 & 6 \\
\hline $\begin{array}{l}\text { Fujisawa } \\
\text { et al. [15]. }\end{array}$ & 1995 & Japan & CC & 222 & NR & NR & DR & 45 & NR & NR & NIDDM & 0.84 & 0.36 & 5 \\
\hline $\begin{array}{c}\text { Tarnow } \\
\text { et al. [16]. }\end{array}$ & 1995 & Denmark & $\mathrm{CC}$ & 155 & $40.9 \pm 9.6$ & $26.7 \pm 7.9$ & PDR & 67 & $42.7 \pm 10.2$ & $25.8 \pm 8.5$ & IDDM & 0.05 & 0.57 & 6 \\
\hline $\begin{array}{l}\text { Nagi } \\
\text { et al. [17]. }\end{array}$ & 1995 & Britain & CC & 271 & $\begin{array}{c}50.6 \pm 14.3 \\
\text { for IDDM } \\
66.8 \pm 10.4 \\
\text { for NIDDM }\end{array}$ & $\begin{array}{c}27(12-66) \\
\text { for IDDM } \\
11(1-36) \\
\text { for NIDDM }\end{array}$ & DR & 376 & $\begin{array}{c}38.3 \pm 14.6 \\
\text { for IDDM } \\
69.5 \pm 11.1 \\
\text { for NIDDM } \\
\text { NA for Healthy } \\
\end{array}$ & $\begin{array}{c}16(1-56) \\
\text { for IDDM } \\
7(1-45) \\
\text { for NIDDM } \\
\text { NA for Healthy } \\
\end{array}$ & $\begin{array}{c}\text { Healthy + } \\
\text { IDDM + } \\
\text { NIDDM }\end{array}$ & 0.71 & 0.52 & 7 \\
\hline Doi et al. [18]. & 1995 & Japan & $\mathrm{CC}$ & 362 & $61.8(30-79)$ & $>10$ & DR & 105 & NA & $\mathrm{NA}$ & Healthy & 0.25 & 0.34 & 4 \\
\hline $\begin{array}{l}\text { Yoshida } \\
\text { et al. [19]. }\end{array}$ & 1996 & Japan & CS & 118 & NA & NA & DR & 50 & NA & NA & NIDDM & 0.59 & 0.31 & 4 \\
\hline $\begin{array}{l}\text { Gutie'rrez } \\
\text { et al. [20]. }\end{array}$ & 1997 & Spain & $\mathrm{CC}$ & 68 & $61.9 \pm 9.1$ & $14.8 \pm 5.7$ & DR & 92 & $59.6 \pm 10.3$ & $12.1 \pm 6.3$ & NIDDM & 0.97 & 0.61 & 6 \\
\hline Liu et al. [21]. & 1997 & China & $\mathrm{CC}$ & 30 & $\mathrm{NA}$ & $\mathrm{NA}$ & DR & 198 & $\begin{array}{l}\text { NA for NDR } \\
34.8 \pm 5.9 \\
\text { for Healthy }\end{array}$ & $\mathrm{NA}$ & $\begin{array}{c}\text { Healthy + } \\
\text { NIDDM }\end{array}$ & 0.92 & 0.27 & 4 \\
\hline Hu et al. [22]. & 1998 & China & CC & 56 & $62.07 \pm 1.21$ & $11.68 \pm 0.91$ & DR & 81 & $\begin{array}{c}56.06 \pm 1.97 \\
\text { for NDR } \\
56.86 \pm 1.46 \\
\text { for Healthy }\end{array}$ & $\begin{array}{c}4.23 \pm 0.47 \\
\text { for NDR }\end{array}$ & $\begin{array}{l}\text { Healthy + } \\
\text { NIDDM }\end{array}$ & 0.02 & 0.35 & 7 \\
\hline $\begin{array}{l}\text { Hanyu } \\
\text { et al. [23]. }\end{array}$ & 1998 & Japan & $\mathrm{CC}$ & 45 & $\begin{array}{c}60.0 \pm 8.8 \\
\text { without DN } \\
56.1 \pm 10.5 \\
\text { with DN }\end{array}$ & $\begin{array}{c}18.2 \pm 5.7 \\
\text { without DN } \\
17.0 \pm 6.0 \\
\text { with DN }\end{array}$ & DR & 57 & $56.4 \pm 5.1$ & NR & Healthy & 0.72 & 0.46 & 6 \\
\hline $\begin{array}{c}\text { Frost } \\
\text { et al. [24]. }\end{array}$ & 1998 & Germany & CS & 79 & $30.1 \pm 6.6$ & $13.1 \pm 8.1$ & DR & 69 & $30.1 \pm 6.6$ & $13.1 \pm 8.1$ & T1DM & 0.87 & 0.67 & 5 \\
\hline $\begin{array}{l}\text { Kimura } \\
\text { et al. [25]. }\end{array}$ & 1998 & Japan & $\mathrm{CC}$ & 114 & NA & NA & PDR & 94 & $43.7 \pm 15.4$ & NR & Healthy & 0.14 & 0.39 & 6 \\
\hline $\begin{array}{l}\text { Rabensteiner } \\
\text { et al. [26]. }\end{array}$ & 1999 & Austria & $\mathrm{CC}$ & 94 & $47.2 \pm 9.9$ & $31.5 \pm 8.2$ & PDR & 81 & $47.7 \pm 11.5$ & $29.7 \pm 8.8$ & T1DM & 0.37 & 0.44 & 6 \\
\hline $\begin{array}{c}\text { Solini } \\
\text { et al. [27]. }\end{array}$ & 1999 & Italy & CS & 21 & NA & NA & DR & 181 & NA & NA & T2DM & 0.11 & 0.67 & 4 \\
\hline $\begin{array}{l}\text { Liao } \\
\text { et al. [28]. }\end{array}$ & 1999 & China & CC & 68 & $\begin{array}{c}51.9 \pm 11.1 \\
\text { for BDR } \\
53.1 \pm 8.8 \\
\text { for PDR }\end{array}$ & $\begin{array}{c}9.35 \pm 3.87 \\
\text { for BDR } \\
9.46 \pm 5.11 \\
\text { for PDR }\end{array}$ & $\mathrm{BDR}+\mathrm{PDR}$ & 76 & $\begin{array}{c}53.2 \pm 8.7 \text { for } \\
\text { NDR } \\
52.3 \pm 9.9 \text { for } \\
\text { Healthy }\end{array}$ & $\begin{array}{c}9.29 \pm 5.17 \\
\text { for NDR }\end{array}$ & $\begin{array}{c}\text { Healthy + } \\
\text { T2DM }\end{array}$ & 0.02 & 0.37 & 7 \\
\hline
\end{tabular}


Table 1. Cont.

\begin{tabular}{|c|c|c|c|c|c|c|c|c|c|c|c|c|c|c|}
\hline \multirow{2}{*}{$\begin{array}{c}\text { Author } \\
\text { (Reference) }\end{array}$} & \multirow{2}{*}{ Year } & \multirow{2}{*}{ Country } & \multirow{2}{*}{ Design } & \multicolumn{4}{|c|}{ Case } & \multicolumn{4}{|c|}{ Control } & \multirow[b]{2}{*}{$\mathrm{HWE}^{\#}$} & \multirow[b]{2}{*}{ MAF * } & \multirow{2}{*}{$\begin{array}{l}\text { NOS } \\
\text { (Stars *) }\end{array}$} \\
\hline & & & & Sample Size & Age (Year) & DM Duration (Year) & Definition & Sample Size & Age (Year) & DM Duration (Year) & Definition & & & \\
\hline $\begin{array}{c}\text { Xiang } \\
\text { et al. [29]. }\end{array}$ & 1999 & China & $\mathrm{CC}$ & 49 & $61.1 \pm 10.5$ & $7.1 \pm 8.2$ & DR & 162 & $\begin{array}{c}53.2 \pm 8.7 \\
\text { for NDR } \\
52.3 \pm 9.9 \\
\text { for Healthy }\end{array}$ & $\begin{array}{c}9.29 \pm 5.17 \\
\text { for NDR }\end{array}$ & $\begin{array}{c}\text { Healthy + } \\
\text { T2DM }\end{array}$ & 0.28 & 0.38 & 7 \\
\hline $\begin{array}{l}\text { Wang } \\
\text { et al. [30]. }\end{array}$ & 1999 & China & CC & 23 & $58.26 \pm 9.57$ & $5.21 \pm 5.7$ & DR & 172 & $\begin{array}{c}59.0 \pm 10.0 \\
\text { for NDR } \\
64.9 \pm 10.0 \\
\text { for Healthy }\end{array}$ & $\begin{array}{l}4.0 \pm 5.1 \\
\text { for NDR }\end{array}$ & $\begin{array}{c}\text { Healthy + } \\
\text { T2DM }\end{array}$ & 0.00 & 0.39 & 7 \\
\hline Liu et al. [31]. & 1999 & China & $\mathrm{CC}$ & 100 & $55(36-90)$ & $8.8(0.5-18)$ & DR & 164 & $\begin{array}{c}53(38-72) \\
\text { for NDR } \\
35 \text { (20-58) } \\
\text { for Healthy }\end{array}$ & NA & $\begin{array}{c}\text { Healthy + } \\
\text { DM }\end{array}$ & 0.21 & 0.40 & 5 \\
\hline $\begin{array}{l}\text { Van Ittersum } \\
\text { et al. [32]. }\end{array}$ & 2000 & $\begin{array}{c}\text { New } \\
\text { Zealand }\end{array}$ & CC & 101 & NA & NA & DR & 151 & NA & NA & IDDM & 0.61 & 0.46 & 4 \\
\hline $\begin{array}{l}\text { Matsumoto } \\
\text { et al. [33]. }\end{array}$ & 2000 & Japan & CC & 120 & $\begin{array}{c}63.2 \pm 10.4 \\
\text { for SDR } \\
56.8 \pm 11.9 \\
\text { for ADR }\end{array}$ & $\begin{array}{c}16.7 \pm 7.6 \\
\text { for SDR } \\
16.2 \pm 9.1 \\
\text { for ADR }\end{array}$ & SDR+ADR & 190 & $\begin{array}{c}58.9 \pm 12.1 \\
\text { for NDR } \\
52.0 \pm 1.0 \\
\text { for Healthy }\end{array}$ & $\begin{array}{l}15.0 \pm 6.6 \\
\text { for NDR }\end{array}$ & $\begin{array}{c}\text { Healthy + } \\
\text { T2DM }\end{array}$ & 0.74 & 0.38 & 7 \\
\hline $\begin{array}{l}\text { Kankova } \\
\text { et al. [34]. }\end{array}$ & 2000 & Czech & $\mathrm{CH}$ & 74 & NA & $\mathrm{NA}$ & PDR & 348 & $\begin{array}{l}63.6 \pm 13.4 \\
\text { for Healthy }\end{array}$ & $\mathrm{NA}$ & $\begin{array}{l}\text { Healthy + } \\
\text { NIDDM }\end{array}$ & 0.19 & 0.52 & 5 \\
\hline $\begin{array}{l}\text { Liao } \\
\text { et al. [35]. }\end{array}$ & 2000 & China & CC & 42 & $\mathrm{NA}$ & $\mathrm{NA}$ & DR & 178 & $\begin{array}{c}54.83 \pm 13.71 \\
\text { for NDR } \\
48.71 \pm 15.12 \\
\text { for Healthy }\end{array}$ & $0.5-30$ for NDR & $\begin{array}{c}\text { Healthy + } \\
\text { T2DM }\end{array}$ & 0.01 & 0.54 & 7 \\
\hline $\begin{array}{c}\text { Yang } \\
\text { et al. [36]. }\end{array}$ & 2000 & China & $\mathrm{CC}$ & 60 & NA & NA & DR & 137 & NA & NA & $\begin{array}{c}\text { Healthy + } \\
\text { NIDDM }\end{array}$ & 0.21 & 0.32 & 4 \\
\hline $\begin{array}{l}\text { Araz } \\
\text { et al. [37]. }\end{array}$ & 2001 & Turkey & $\mathrm{CS} / \mathrm{CC}$ & 120 & $55.0 \pm 8.0$ & $11.2 \pm 6.5$ & DR & 257 & $\begin{array}{c}51.0 \pm 9.0 \text { for } \\
\text { NDR } \\
\text { NA for Healthy }\end{array}$ & $\begin{array}{l}5.2 \pm 5.1 \\
\text { for NDR }\end{array}$ & $\begin{array}{c}\text { Healthy + } \\
\text { T2DM }\end{array}$ & 0.98 & 0.60 & 7 \\
\hline $\begin{array}{l}\text { Viswanathan } \\
\text { et al. [38]. }\end{array}$ & 2001 & India & $\mathrm{CC}$ & 86 & $56.7+8.9$ & $13.4+6.9$ & DR & 23 & $56.7+9.3$ & $13.2+5.1$ & T2DM & 0.01 & 0.46 & 6 \\
\hline $\begin{array}{l}\text { Petrovic } \\
\text { et al. [39]. }\end{array}$ & 2003 & Slovenia & $\mathrm{CC}$ & 124 & $65.6 \pm 9.7$ & $18.7 \pm 9.1$ & DR & 80 & $71.3 \pm 7.0$ & $16.8 \pm 6.8$ & T2DM & 0.07 & 0.51 & 6 \\
\hline Ha et al. [40]. & 2003 & Korea & CS & 180 & NA & $\mathrm{NA}$ & DR & 59 & $\mathrm{NA}$ & NA & T2DM & 0.07 & 0.37 & 4 \\
\hline $\begin{array}{c}\text { Crook } \\
\text { et al. [41]. }\end{array}$ & 2003 & USA & $\mathrm{CH}$ & 46 & NA & NA & DR & 10 & NA & NA & T2DM & 0.24 & 0.80 & 4 \\
\hline $\begin{array}{l}\text { Agardh } \\
\text { et al. [42]. }\end{array}$ & 2003 & USA & $\mathrm{CC}$ & 24 & 32 (24-37) & $23(16-31)$ & SDR & 24 & $28.5(22-57)$ & $19.5(10-56)$ & T1DM & 0.74 & 0.56 & 6 \\
\hline Xu et al. [43]. & 2003 & China & $\mathrm{CC}$ & 58 & $62 \pm 10$ & $8 \pm 6$ & DR & 142 & $\begin{array}{c}60 \pm 12 \text { for } \\
\text { NDR } \\
59 \pm 12 \text { for } \\
\text { Healthy }\end{array}$ & $8 \pm 7$ for $\mathrm{NDR}$ & $\begin{array}{c}\text { Healthy + } \\
\text { T2DM }\end{array}$ & 0.03 & 0.35 & 7 \\
\hline
\end{tabular}


Table 1. Cont

\begin{tabular}{|c|c|c|c|c|c|c|c|c|c|c|c|c|c|c|}
\hline \multirow{2}{*}{$\begin{array}{c}\text { Author } \\
\text { (Reference) }\end{array}$} & \multirow{2}{*}{ Year } & \multirow{2}{*}{ Country } & \multirow{2}{*}{ Design } & \multicolumn{4}{|c|}{ Case } & \multicolumn{4}{|c|}{ Control } & \multirow{2}{*}{$\mathrm{HWE}^{\#}$} & \multirow{2}{*}{$\mathrm{MAF}^{*}$} & \multirow{2}{*}{$\begin{array}{c}\text { NOS } \\
\text { (Stars * }\end{array}$} \\
\hline & & & & Sample Size & Age (Year) & DM Duration (Year) & Definition & Sample Size & Age (Year) & DM Duration (Year) & Definition & & & \\
\hline $\begin{array}{l}\text { Thomas } \\
\text { et al. [55]. }\end{array}$ & 2003 & China/Asia & $\mathrm{CC}$ & 326 & $59.8 \pm 11.4$ & $6.3(5.6-7.0)$ & DR & 501 & $\begin{array}{l}60.4 \pm 9.3 \text { for } \\
\text { T2DM }\end{array}$ & $6.0(5.6-6.3)$ & T2DM & 0.38 & 0.33 & 6 \\
\hline Wu et al. [44]. & 2004 & China & $\mathrm{CH}$ & 90 & $\begin{array}{l}30.5 \pm 4.3 \\
\text { for T1DR } \\
60.2 \pm 8.3 \\
\text { for T2DR }\end{array}$ & $\begin{array}{l}11.8 \pm 2.4 \\
\text { for T1DR } \\
15.1 \pm 4.7 \\
\text { for T2DR }\end{array}$ & DR & 294 & $\begin{array}{c}36.8 \pm 6.6 \\
\text { for T1DM } \\
65.2 \pm 3.2 \\
\text { for T2DM MI } \\
59.5 \pm 1.2 \\
\text { for T2DM NMI }\end{array}$ & $\begin{array}{c}24.3 \pm 9.8 \\
\text { for T1DM } \\
15.1 \pm 5.0 \\
\text { for T2DM MI } \\
12.3 \pm 3.3 \\
\text { for T2DM NMI }\end{array}$ & $\begin{array}{l}\text { T1DM + } \\
\text { T2DM }\end{array}$ & 0.22 & 0.57 & 8 \\
\hline $\begin{array}{l}\text { Liao et al. } \\
\text { [45]. }\end{array}$ & 2004 & China & CC & 44 & NA & NA & $\mathrm{BDR}+\mathrm{PDR}$ & 21 & NA & NA & T2DM & 0.16 & 0.40 & 4 \\
\hline $\begin{array}{l}\text { Degirmenci } \\
\text { et al. [46]. }\end{array}$ & 2005 & Turkey & CC & 57 & NA & NA & DR & 83 & NA & $\mathrm{NA}$ & T2DM & 0.61 & 0.54 & 4 \\
\hline $\begin{array}{l}\text { Chen } \\
\text { et al. [47]. }\end{array}$ & 2005 & China & CC & 27 & $58.39 \pm 9.47$ & NA & DR & 319 & $\begin{array}{c}55.43 \pm 8.31 \text { for } \\
\text { NDR } \\
\text { NA for Healthy }\end{array}$ & NA & $\begin{array}{c}\text { Healthy + } \\
\text { T2DM }\end{array}$ & 0.39 & 0.63 & 5 \\
\hline Lee et al. [48]. & 2006 & Korea & CC & 130 & $53.1 \pm 12.3$ & $11.4 \pm 3.7$ & DR & 174 & $53.7 \pm 12.9$ & $9.4 \pm 2.8$ & T2DM & 0.01 & 0.42 & 6 \\
\hline $\begin{array}{l}\text { Liang } \\
\text { et al. [49]. }\end{array}$ & 2006 & China & CC & 82 & $63.41 \pm 11.22$ & $8.34 \pm 6.36$ & DR & 153 & $\begin{array}{c}62.98 \pm 11.87 \\
\text { for NDR } \\
65.31 \pm 9.77 \\
\text { for Healthy }\end{array}$ & $\begin{array}{l}4.91 \pm 4.76 \\
\text { for NDR }\end{array}$ & $\begin{array}{c}\text { Healthy + } \\
\text { T2DM }\end{array}$ & 0.54 & 0.32 & 7 \\
\hline $\begin{array}{l}\text { Nikzamir } \\
\text { et al. [50]. }\end{array}$ & 2010 & Iran & CC & 178 & $59.0 \pm 8.7$ & $13(4-30)$ & DR & 206 & $59.5 \pm 8.2$ & $11(1-30)$ & T2DM & 0.29 & 0.46 & 6 \\
\hline Li et al. [51]. & 2013 & China & CC & 207 & $62.4 \pm 7.8$ & $14.6 \pm 7.5$ & DR & 302 & $\begin{array}{c}59.5 \pm 8.2 \\
\text { for NDR } \\
75.5 \pm 2.8 \\
\text { for Healthy }\end{array}$ & $\begin{array}{c}15.0 \pm 4.3 \\
\text { for NDR }\end{array}$ & $\begin{array}{c}\text { Healthy + } \\
\text { T2DM }\end{array}$ & 0.02 & 0.50 & 7 \\
\hline $\begin{array}{l}\text { Narne } \\
\text { et al. [54]. }\end{array}$ & 2016 & India & $\mathrm{CC}$ & 149 & $52.7 \pm 7.3$ & $14.7 \pm 4.7$ & DR & 162 & $53.4 \pm 5.4$ & $15.9 \pm 5.6$ & T2DM & 0.05 & 0.40 & 6 \\
\hline
\end{tabular}

The reference was referred to the reference numbers in this study; \# Hardy-Weinberg equilibrium (HWE) test and * the minor allele frequency (MAF) were calculated in the control group for each study; NR, not reported; NA, not available; CC, case-control; CS, cross-sectional; $\mathrm{CH}$ cohort; DR, diabetes retinopathy; BDR, background diabetes retinopathy; diabetes mellitus; T1DM, type 1 diabetes mellitus; T2DM, type 2 diabetes mellitus; IDDM, insulin dependent diabetes mellitus; NIDDM, non-insulin dependent diabetes mellitus; MI,

myocardial infarct; NMI, non-myocardial infract. 


\section{Results}

\subsection{Literature Search}

The study selection process is shown in Figure 1. A total of 660 articles (PubMed 572, CNKI 88) were identified from the databases, and 0 duplicates were excluded, using EndNote (X7) (Thomson ResearchSoft, Stamford, CT, USA). In addition, 581 articles were excluded, based on a review of the titles and abstracts, and 79 full-text articles were assessed for eligibility; 37 articles were excluded due to various reasons, such as being review articles or case reports, being written in languages other than English or Chinese, or could not provide each genotype frequency or other sufficient information for extraction of data. Finally, a total of 40 [14-51,54,55] articles were included in this meta-analysis.

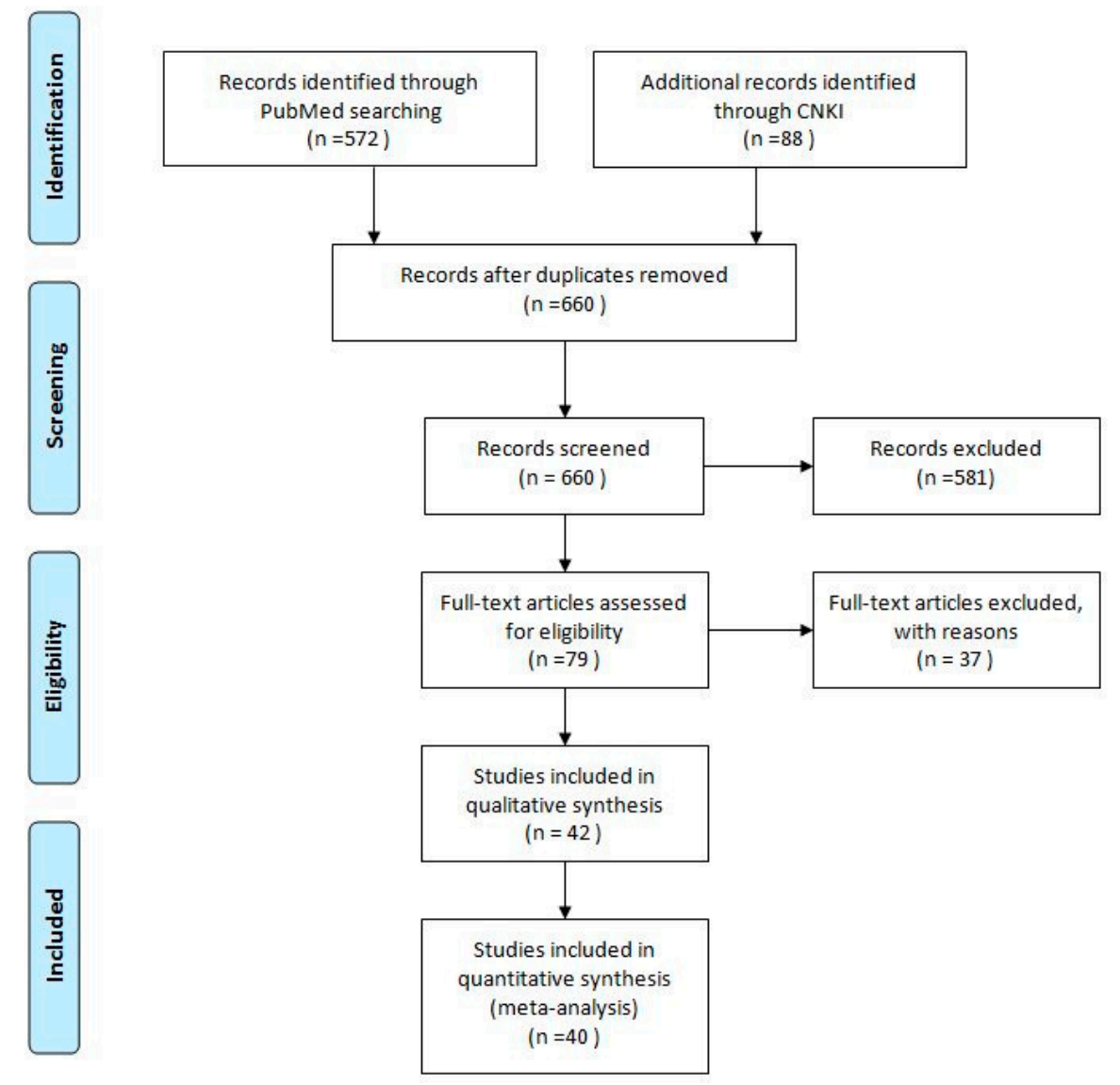

Figure 1. Flow chart of the literature search.

\subsection{Eligible Studies and Study Characteristics}

The selected study characteristics from the studies included in the meta-analysis are provided in Table 1, and the details on ACE I/D polymorphism allele/genotype prevalence are shown in Table 2. For 40 studies, 8 studies (7 Non-Asian, 1 Asian) involved cases with T1DM, 33 (9 Non-Asian, 24 Asian) with T2DM, and 1 study ([21])with un-defined DM type (1 Asian study with 100 cases and 164 controls). It is worth emphasizing that 2 studies $([17,44])$ involved both T1DM and T2DM. The studies on T1DM Non-Asians contributed 599 cases and 614 control subjects, while the Asian studies included 33 cases and 104 control subjects. Among the T2DM studies, studies involving Non-Asians contributed 865 cases and 1541 control subjects, while the Asian studies included 2655 cases and 3659 control subjects. Thirty-three studies were case-control study design, 4 studies were cross-sectional study design, and 3 studies were cohort study design. 
Table 2. The details on ACE I/D (angiotensin-converting enzyme insertion/deletion) polymorphism allele/genotype prevalence.

\begin{tabular}{|c|c|c|c|c|c|c|c|c|c|c|}
\hline \multirow{3}{*}{ Author (Reference) } & \multicolumn{6}{|c|}{ Prevalence of $A C E$ I/D Genotype } & \multicolumn{4}{|c|}{ Prevalence of Allele Frequency } \\
\hline & \multicolumn{2}{|c|}{ II } & \multicolumn{2}{|c|}{ ID } & \multicolumn{2}{|c|}{ DD } & \multicolumn{2}{|c|}{ I } & \multicolumn{2}{|c|}{ D } \\
\hline & Case & Control & Case & Control & Case & Control & Case & Control & Case & Control \\
\hline Marre et al. [14]. & 8 & 3 & 28 & 17 & 16 & 12 & 44 & 23 & 60 & 41 \\
\hline Fujisawa et al. [15]. & 87 & 19 & 102 & 20 & 33 & 6 & 276 & 58 & 168 & 32 \\
\hline Tarnow et al. [16]. & 29 & 16 & 74 & 25 & 52 & 26 & 132 & 57 & 178 & 77 \\
\hline Nagi et al. [17]. & 74 & 88 & 120 & 184 & 77 & 104 & 268 & 360 & 274 & 392 \\
\hline Doi et al. [18]. & 132 & 48 & 179 & 42 & 51 & 15 & 443 & 138 & 281 & 72 \\
\hline Yoshida et al. [19]. & 45 & 23 & 51 & 23 & 22 & 4 & 141 & 69 & 95 & 31 \\
\hline Gutie'rrez et al. [20]. & 6 & 14 & 30 & 44 & 32 & 34 & 42 & 72 & 94 & 112 \\
\hline Liu et al. [21]. & 10 & 105 & 8 & 78 & 12 & 15 & 28 & 288 & 32 & 108 \\
\hline Hu et al. [22]. & 29 & 39 & 15 & 27 & 12 & 15 & 73 & 105 & 39 & 57 \\
\hline Hanyu et al. [23]. & 21 & 17 & 18 & 27 & 6 & 13 & 60 & 61 & 30 & 53 \\
\hline Frost et al. [24]. & 23 & 8 & 25 & 30 & 31 & 31 & 71 & 46 & 87 & 92 \\
\hline Kimura et al. [25]. & 48 & 38 & 47 & 38 & 19 & 18 & 143 & 114 & 85 & 74 \\
\hline Rabensteiner et al. [26]. & 11 & 23 & 46 & 44 & 37 & 14 & 68 & 90 & 120 & 72 \\
\hline Solini et al. [27]. & 4 & 25 & 16 & 71 & 1 & 85 & 24 & 121 & 18 & 241 \\
\hline Liao et al. [28]. & 33 & 35 & 21 & 26 & 14 & 15 & 87 & 96 & 49 & 56 \\
\hline Xiang et al. [29]. & 12 & 65 & 23 & 70 & 14 & 27 & 47 & 200 & 51 & 124 \\
\hline Wang et al. [30]. & 9 & 75 & 8 & 61 & 6 & 36 & 26 & 211 & 20 & 133 \\
\hline Liu et al. [31]. & 33 & 63 & 38 & 71 & 29 & 30 & 104 & 197 & 96 & 131 \\
\hline Van Ittersum et al. [32]. & 29 & 45 & 47 & 72 & 25 & 34 & 105 & 162 & 97 & 140 \\
\hline Matsumoto et al. [33]. & 41 & 75 & 53 & 87 & 26 & 28 & 135 & 237 & 105 & 143 \\
\hline Kankova et al. [34]. & 14 & 75 & 39 & 186 & 21 & 87 & 67 & 336 & 81 & 360 \\
\hline Liao et al. [35]. & 11 & 46 & 18 & 72 & 13 & 60 & 40 & 164 & 44 & 192 \\
\hline Yang et al. [36]. & 22 & 60 & 14 & 66 & 24 & 11 & 58 & 186 & 62 & 88 \\
\hline Araz et al. [37]. & 20 & 42 & 62 & 124 & 38 & 91 & 102 & 208 & 138 & 306 \\
\hline Viswanathan et al. [38]. & 17 & 10 & 45 & 5 & 24 & 8 & 79 & 25 & 93 & 21 \\
\hline Petrovic et al. [39]. & 28 & 23 & 63 & 32 & 33 & 25 & 119 & 78 & 129 & 82 \\
\hline Ha et al. [40]. & 48 & 20 & 85 & 34 & 47 & 5 & 181 & 74 & 179 & 44 \\
\hline Crook et al. [41]. & 5 & 1 & 27 & 2 & 14 & 7 & 37 & 4 & 55 & 16 \\
\hline Agardh et al. [42]. & 4 & 5 & 11 & 11 & 9 & 8 & 19 & 21 & 29 & 27 \\
\hline Xu et al. [43]. & 11 & 66 & 31 & 53 & 16 & 23 & 53 & 185 & 63 & 99 \\
\hline Thomas et al. [55]. & 157 & 231 & 129 & 212 & 40 & 58 & 443 & 674 & 209 & 328 \\
\hline Wu et al. [44]. & 11 & 60 & 45 & 134 & 34 & 100 & 67 & 254 & 113 & 334 \\
\hline Liao et al. [45]. & 19 & 9 & 16 & 7 & 9 & 5 & 54 & 25 & 34 & 17 \\
\hline Degirmenci et al. [46]. & 6 & 19 & 34 & 39 & 17 & 25 & 46 & 77 & 68 & 89 \\
\hline Chen et al. [47]. & 3 & 39 & 5 & 155 & 19 & 125 & 11 & 233 & 43 & 405 \\
\hline Lee et al. [48]. & 47 & 67 & 69 & 68 & 14 & 39 & 163 & 202 & 97 & 146 \\
\hline Liang et al. [49]. & 26 & 73 & 36 & 63 & 20 & 17 & 88 & 209 & 76 & 97 \\
\hline Nikzamir et al. [50]. & 47 & 56 & 73 & 110 & 58 & 40 & 167 & 222 & 189 & 190 \\
\hline Li et al. [51]. & 52 & 64 & 120 & 172 & 35 & 66 & 224 & 300 & 190 & 304 \\
\hline Narne et al. [54]. & 46 & 64 & 76 & 66 & 27 & 32 & 168 & 194 & 130 & 130 \\
\hline Total & 1278 & 1854 & 1947 & 2668 & 1027 & 1394 & 4503 & 63,762 & 4001 & 5456 \\
\hline
\end{tabular}

\subsection{Summary Statistics}

Data from 40 articles that investigated the association between the ACE I/D polymorphism and DR risk were included in the meta-analysis. The overall frequency (\%) of minor D allele frequency (MAF) was $0.47 / 0.46$ for cases and controls. The frequency of the MAF for each study polymorphism on controls is shown in Table 1. All studies suggested that the genotypes distribution in controls was consistent with the Hardy-Weinberg equilibrium except for 8 studies ([22,28,30,35,38,43,48,51]), indicating genotyping errors and/or population stratification [59]; therefore, a sensitivity analysis was performed by excluding these studies.

\subsection{Main Results, Stratification, and Sensitivity Analyses}

The estimation of the relationship of ACE I/D polymorphism with DR is presented in Table 3. Figure 2 shows the overall effect for the relationship between the polymorphism and the DR risk in dominant model. 
Table 3. Summary ORs and heterogeneity results for associations between the ACE I/D polymorphism and DR (diabetic retinopathy).

\begin{tabular}{|c|c|c|c|c|c|c|c|}
\hline Genetic Model & Group & Sensitivity $\#$ & Studies & OR & $95 \%$ CI & $p^{*}$ & $I^{2}(\%)$ \\
\hline \multirow{18}{*}{ ID vs. II } & \multirow{2}{*}{ All studies } & All & 40 & 1.14 & $1.00-1.30$ & 0.02 & 33.8 \\
\hline & & Sensitivity & 32 & 1.08 & $0.97-1.21$ & 0.13 & 22.60 \\
\hline & \multirow{2}{*}{ Non-Asian } & All & 15 & 1.04 & $0.86-1.25$ & 0.09 & 35.30 \\
\hline & & Sensitivity & 15 & 1.04 & $0.86-1.25$ & 0.09 & 35.30 \\
\hline & \multirow{2}{*}{ Asian } & All & 25 & 1.14 & $1.01-1.29$ & 0.05 & 34.50 \\
\hline & & Sensitivity & 17 & 1.11 & $0.96-1.29$ & 0.32 & 11.50 \\
\hline & \multirow{2}{*}{ TIDM } & All & 8 & 1.00 & $0.64-1.56$ & 0.05 & 50.30 \\
\hline & & Sensitivity & 8 & 1.00 & $0.64-1.56$ & 0.05 & 50.30 \\
\hline & \multirow[b]{2}{*}{ T2DM } & All & 33 & 1.13 & $1.00-1.24$ & 0.05 & 31.20 \\
\hline & & Sensitivity & 26 & 1.07 & $1.00-1.21$ & 0.30 & 11.40 \\
\hline & \multirow{2}{*}{ Non-Asian with T1DM } & All & 7 & 0.98 & $0.84-1.14$ & 0.04 & 55.40 \\
\hline & & Sensitivity & 7 & 0.98 & $0.84-1.14$ & 0.04 & 55.40 \\
\hline & \multirow{2}{*}{ Non-Asian with T2DM } & All & 9 & 1.03 & $0.96-1.10$ & 0.49 & 0.00 \\
\hline & & Sensitivity & 9 & 1.03 & $0.96-1.10$ & 0.49 & 0.00 \\
\hline & \multirow{2}{*}{ Asian with T1DM } & All & 1 & 1.13 & $0.87-1.46$ & NA & NA \\
\hline & & Sensitivity & 1 & 1.13 & $0.87-1.46$ & NA & NA \\
\hline & \multirow[b]{2}{*}{ Asian with T2DM } & All & 24 & 1.14 & $1.01-1.30$ & 0.05 & 36.10 \\
\hline & & Sensitivity & 16 & 1.11 & $1.00-1.29$ & 0.29 & 13.90 \\
\hline \multirow{18}{*}{ DD vs. II } & \multirow{2}{*}{ All studies } & All & 40 & 1.38 & $1.11-1.71$ & 0.00 & 62.3 \\
\hline & & Sensitivity & 32 & 1.46 & $1.15-1.87$ & 0.00 & 62.20 \\
\hline & \multirow{2}{*}{ Non-Asian } & All & 15 & 1.14 & $0.81-1.60$ & 0.01 & 55.50 \\
\hline & & Sensitivity & 15 & 1.14 & $0.81-1.60$ & 0.01 & 55.50 \\
\hline & \multirow{2}{*}{ Asian } & All & 25 & 1.54 & $1.16-2.04$ & 0.00 & 65.30 \\
\hline & & Sensitivity & 17 & 1.80 & $1.30-2.51$ & 0.00 & 63.20 \\
\hline & \multirow{2}{*}{ TIDM } & All & 8 & 1.08 & $0.63-1.87$ & 0.01 & 61.70 \\
\hline & & Sensitivity & 8 & 1.08 & $0.63-1.87$ & 0.01 & 61.70 \\
\hline & \multirow{2}{*}{ T2DM } & All & 33 & 1.39 & $1.10-1.74$ & 0.00 & 61.80 \\
\hline & & Sensitivity & 26 & 1.58 & $1.20-2.07$ & 0.00 & 66.20 \\
\hline & \multirow{2}{*}{ Non-Asian with T1DM } & All & 7 & 1.09 & $0.92-1.30$ & 0.09 & 44.90 \\
\hline & & Sensitivity & 7 & 1.09 & $0.92-1.30$ & 0.09 & 44.90 \\
\hline & \multirow{2}{*}{ Non-Asian with T2DM } & All & 9 & 1.06 & $0.96-1.18$ & 0.26 & 20.20 \\
\hline & & Sensitivity & 9 & 1.06 & $0.96-1.18$ & 0.26 & 20.20 \\
\hline & & All & 1 & 0.99 & $0.64-1.53$ & NA & NA \\
\hline & Asian with IIDM & Sensitivity & 1 & 0.99 & $0.64-1.53$ & NA & NA \\
\hline & & All & 24 & 1.54 & $1.14-2.08$ & 0.00 & 66.70 \\
\hline & Asian with T2DM & Sensitivity & 16 & 1.83 & $1.27-2.63$ & 0.00 & 65.80 \\
\hline & & All & 40 & 1.17 & $1.05-1.30$ & 0 & 64.7 \\
\hline & All studies & Sensitivity & 32 & 1.19 & $1.05-1.35$ & 0.00 & 65.40 \\
\hline & & All & 15 & 1.02 & $0.86-1.22$ & 0.00 & 62.10 \\
\hline & Non-Asian & Sensitivity & 15 & 1.02 & $0.86-1.22$ & 0.00 & 62.10 \\
\hline & & All & 25 & 1.26 & $1.10-1.45$ & 0.00 & 65.40 \\
\hline & Asian & Sensitivity & 17 & 1.35 & $1.15-1.59$ & 0.00 & 64.00 \\
\hline & & All & 8 & 1.03 & $0.78-1.34$ & 0.01 & 61.00 \\
\hline Allele contrast & IIDIVI & Sensitivity & 8 & 1.03 & $0.78-1.34$ & 0.01 & 61.00 \\
\hline & & All & 33 & 1.17 & $1.04-1.32$ & 0.00 & 64.90 \\
\hline & I 2DM & Sensitivity & 26 & 1.22 & $1.06-1.40$ & 0.00 & 66.50 \\
\hline & & All & 7 & 1.02 & 0.89-1.16 & 0.01 & 65.40 \\
\hline & Non-Asian with T1DM & Sensitivity & 7 & 1.02 & $0.89-1.16$ & 0.01 & 65.40 \\
\hline & & All & 9 & 1.01 & $0.92-1.10$ & 0.02 & 54.80 \\
\hline & Non-Asian with 1 2DMI & Sensitivity & 9 & 1.01 & $0.92-1.10$ & 0.02 & 54.80 \\
\hline & & All & 1 & 0.96 & $0.76-1.23$ & NA & NA \\
\hline & Asian with IIDM & Sensitivity & 1 & 0.96 & $0.76-1.23$ & NA & NA \\
\hline & & All & 24 & 1.26 & $1.09-1.47$ & 0.00 & 66.90 \\
\hline & Asian with I2DM & Sensitivity & 16 & 1.36 & $1.14-1.63$ & 0.00 & 66.30 \\
\hline
\end{tabular}


Table 3. Cont.

\begin{tabular}{|c|c|c|c|c|c|c|c|}
\hline Genetic Model & Group & Sensitivity ${ }^{\#}$ & Studies & OR & $95 \% \mathrm{CI}$ & $p^{*}$ & $I^{2}(\%)$ \\
\hline \multirow{18}{*}{ Recessive model } & \multirow{2}{*}{ All studies } & All & 40 & 1.24 & $1.02-1.51$ & 0 & 67.6 \\
\hline & & Sensitivity & 32 & 1.33 & $1.07-1.66$ & 0.00 & 69.20 \\
\hline & \multirow{2}{*}{ Non-Asian } & All & 15 & 1.03 & $0.79-1.35$ & 0.00 & 59.70 \\
\hline & & Sensitivity & 15 & 1.03 & $0.79-1.35$ & 0.00 & 59.70 \\
\hline & \multirow{2}{*}{ Asian } & All & 25 & 1.42 & $1.08-1.85$ & 0.00 & 71.10 \\
\hline & & Sensitivity & 17 & 1.73 & $1.24-2.41$ & 0.00 & 71.90 \\
\hline & \multirow{2}{*}{ TIDM } & All & 8 & 1.09 & $0.86-1.39$ & 0.09 & 43.20 \\
\hline & & Sensitivity & 8 & 1.09 & $0.86-1.39$ & 0.09 & 43.20 \\
\hline & \multirow{2}{*}{ T2DM } & All & 33 & 1.24 & $1.01-1.54$ & 0.00 & 69.50 \\
\hline & & Sensitivity & 26 & 1.36 & $1.06-1.74$ & 0.00 & 71.90 \\
\hline & \multirow{2}{*}{ Non-Asian with T1DM } & All & 7 & 1.09 & $0.92-1.30$ & 0.09 & 44.90 \\
\hline & & Sensitivity & 7 & 1.09 & $0.92-1.30$ & 0.09 & 44.90 \\
\hline & \multirow{2}{*}{ Non-Asian with T2DM } & All & 9 & 1.00 & $0.75-1.25$ & 0.00 & 67.20 \\
\hline & & Sensitivity & 9 & 1.00 & $0.75-1.25$ & 0.00 & 67.20 \\
\hline & \multirow{2}{*}{ Asian with T1DM } & All & 1 & 0.76 & $0.42-1.42$ & NA & NA \\
\hline & & Sensitivity & 1 & 0.76 & $0.42-1.42$ & NA & NA \\
\hline & \multirow{2}{*}{ Asian with T2DM } & All & 24 & 1.42 & $1.07-1.88$ & 0.00 & 71.80 \\
\hline & & Sensitivity & 16 & 1.76 & $1.23-2.51$ & 0.00 & 72.90 \\
\hline \multirow{18}{*}{$\begin{array}{c}\text { Dominant } \\
\text { model }\end{array}$} & \multirow{2}{*}{ All studies } & All & 40 & 1.21 & $1.06-1.38$ & 0.01 & 37.8 \\
\hline & & Sensitivity & 32 & 1.17 & $1.06-1.31$ & 0.05 & 30.50 \\
\hline & \multirow{2}{*}{ Non-Asian } & All & 15 & 1.15 & $0.97-1.37$ & 0.18 & 25.30 \\
\hline & & Sensitivity & 15 & 1.15 & $0.97-1.37$ & 0.18 & 25.30 \\
\hline & \multirow{2}{*}{ Asian } & All & 25 & 1.26 & $1.08-1.47$ & 0.03 & 37.60 \\
\hline & & Sensitivity & 17 & 1.25 & $1.09-1.42$ & 0.02 & 19.80 \\
\hline & \multirow{2}{*}{ TIDM } & All & 8 & 1.03 & $0.66-1.61$ & 0.02 & 57.30 \\
\hline & & Sensitivity & 8 & 1.03 & $0.66-1.61$ & 0.02 & 57.30 \\
\hline & \multirow{2}{*}{ T2DM } & All & 33 & 1.19 & $1.05-1.36$ & 0.04 & 32.20 \\
\hline & & Sensitivity & 26 & 1.16 & $1.04-1.29$ & 0.20 & 18.60 \\
\hline & \multirow{2}{*}{ Non-Asian with T1DM } & All & 7 & 1.00 & $0.90-1.11$ & 0.01 & 63.00 \\
\hline & & Sensitivity & 7 & 1.00 & $0.90-1.11$ & 0.01 & 63.00 \\
\hline & \multirow{2}{*}{ Non-Asian with T2DM } & All & 9 & 1.02 & $0.98-1.07$ & 0.67 & 0.00 \\
\hline & & Sensitivity & 9 & 1.02 & $0.98-1.07$ & 0.67 & 0.00 \\
\hline & \multirow{2}{*}{ Asian with T1DM } & All & 1 & 1.05 & $0.89-1.25$ & NA & NA \\
\hline & & Sensitivity & 1 & 1.05 & $0.89-1.25$ & NA & NA \\
\hline & \multirow{2}{*}{ Asian with T2DM } & All & 24 & 1.26 & $1.07-1.49$ & 0.02 & 40.20 \\
\hline & & Sensitivity & 16 & 1.24 & $1.08-1.43$ & 0.17 & 25.00 \\
\hline
\end{tabular}

\# Sensitivity analysis for HWE; * test for heterogeneity; random-effects model was used when $p$ value for heterogeneity test $<0.05$ and $I^{2}>50 \%$; otherwise, fixed-effects model was used.

As shown in Table 3, the overall analysis found a significant association between the ACE I/D polymorphism and the risk of DR for all genetic models (ID vs. II: OR $=1.14,95 \%$ CI: $1.00-1.30$; DD vs. II: OR $=1.38,95 \%$ CI: 1.11-1.71; Allele contrast: $\mathrm{OR}=1.17,95 \%$ CI: $1.05-1.30$; recessive model: $\mathrm{OR}=1.24,95 \%$ CI: $1.02-1.51$ and dominant model: $\mathrm{OR}=1.21,95 \%$ CI: 1.06-1.38, respectively).

In a stratified analysis by ethnicity and DM type, we further detected that the Asian group, T2DM group, and Asian group with T2DM all showed significant associations for all genetic models (ID vs. II: $\mathrm{OR}=1.14,95 \% \mathrm{CI}: 1.01-1.29$ for the Asian group, OR $=1.13,95 \% \mathrm{CI}: 1.00-1.24$ for the T2DM group and $\mathrm{OR}=1.14,95 \%$ CI: 1.01-1.30 for the Asian group with T2DM, respectively; DD vs. II: OR $=1.54$, 95\% CI: $1.16-2.04$ for the Asian group, OR $=1.39,95 \%$ CI: $1.10-1.74$ for the T2DM group and OR $=1.54$, 95\% CI: 1.14-2.08 for the Asian group with T2DM, respectively; Allele contrast: OR $=1.26,95 \% \mathrm{CI}$ : $1.10-1.45$ for the Asian group, OR $=1.17,95 \%$ CI: $1.04-1.32$ for the T2DM group and OR $=1.26,95 \%$ CI: 1.09-1.47 for the Asian group with T2DM, respectively; recessive model: OR $=1.42,95 \%$ CI: $1.08-1.85$ for the Asian group, OR = 1.24, 95\% CI: 1.01-1.54 for the T2DM group and OR $=1.42,95 \%$ CI: $1.07-1.88$ for the Asian group with T2DM, respectively and dominant model: OR $=1.26,95 \%$ CI: $1.08-1.47$ for 
the Asian group, $\mathrm{OR}=1.19,95 \% \mathrm{CI}$ : $1.05-1.36$ for the T2DM group and OR $=1.26,95 \%$ CI: $1.07-1.49$ for the Asian group with T2DM, respectively). However, we did not find any significant effects for different genetic models in other subgroup. Further sensitivity analysis for HWE did not alter the pattern of results in both overall analysis and subgroup analysis.

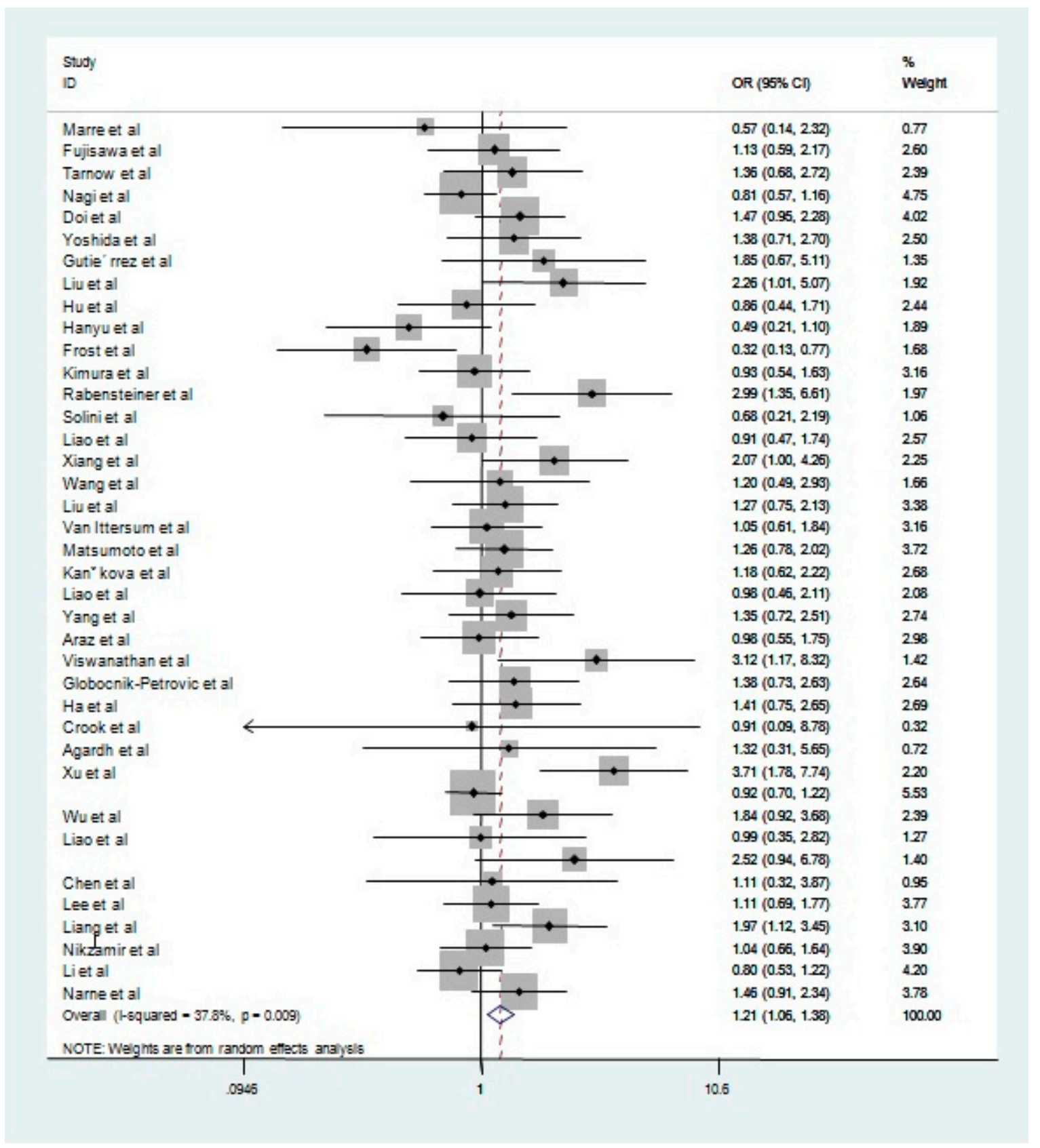

Figure 2. ORs (log scale) of DR associated with ACE I/D polymorphism for dominant genetic model. The graph shows individual and pooled estimates for all studies.

\subsection{Source of Heterogeneity and Publication Bias}

From Table 3, we found that the heterogeneity between studies was observed in overall comparisons as well as subgroup analyses. We estimated the source of heterogeneity in both dominant and recessive genetic models of the variant allele by ethnicity (Asian or Non-Asian), DM type (T1DM or T2DM), HWE (in HWE or not), and study design (case-control, cross-sectional, or cohort study design) 
by meta-regression analyses. It revealed that none of these four factors could influence significant between-study heterogeneity in genetic models for ACE I/D polymorphism: ethnicity ( $p=0.78$ for dominant model and $p=0.39$ for recessive model), DM type ( $p=0.59$ for dominant model and $p=0.9$ for recessive model), HWE ( $p=0.26$ for dominant model and $p=0.77$ for recessive model), and study design ( $p=0.06$ for dominant model and $p=0.24$ for recessive model).

The potential presence of publication bias was estimated by using a funnel plot of the evaluation of log-odds ratio for the genotype DD+ ID versus II against the reciprocal of its standard error (Figure 3). As shown, we failed to find any significant funnel asymmetry to indicate publication bias. We further used the Egger regression asymmetry test and the Begg adjusted rank correlation test to estimate the publication bias of literatures included in the meta-analysis. As shown in Table 4, no publication bias was found for polymorphism and risk of DR in genetic models.

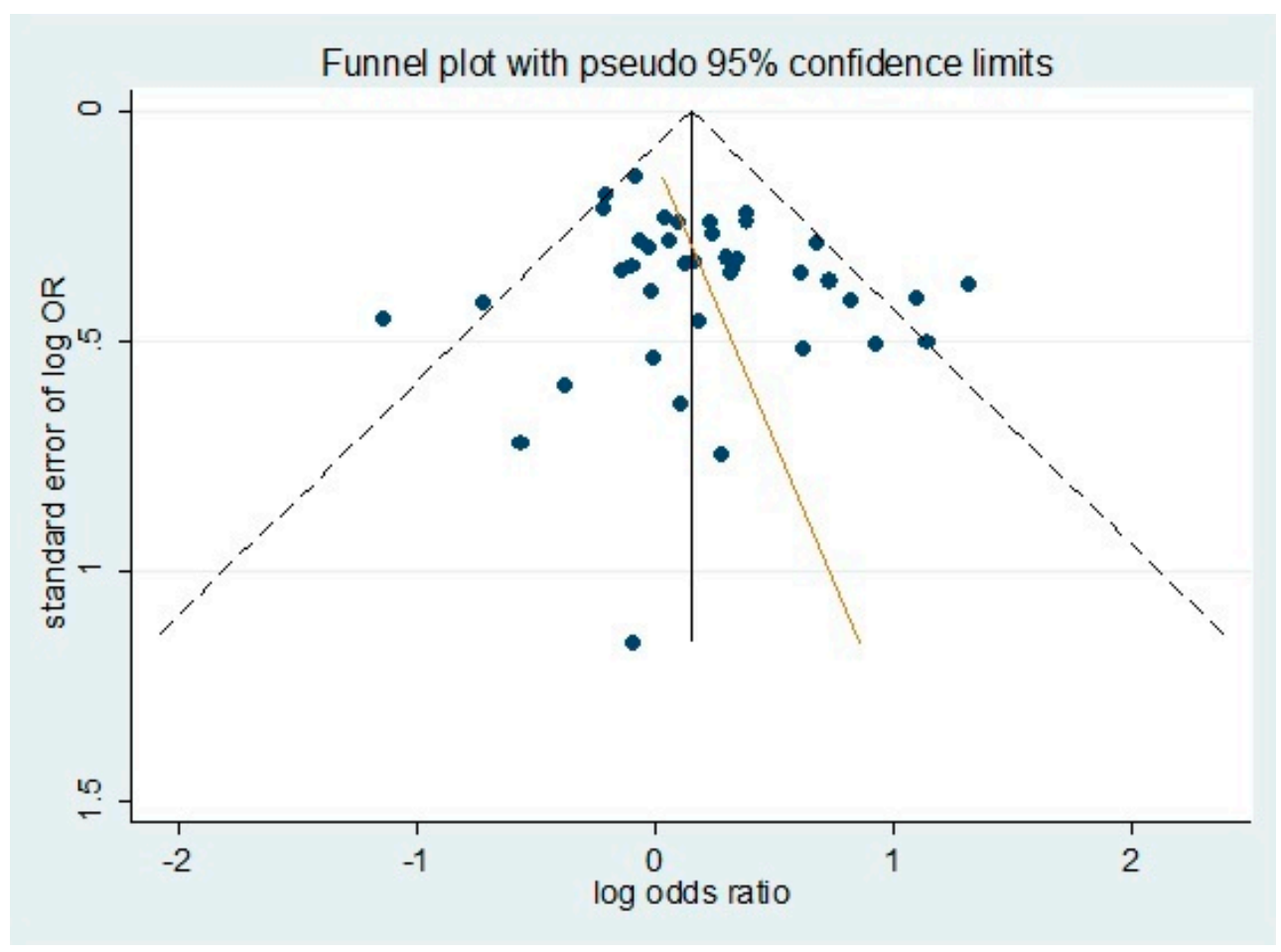

Figure 3. Evaluation of publication bias using funnel plots. Plots are shown for all studies.

Table 4. The results of publication bias test by Egger and Begg test.

\begin{tabular}{ccccc}
\hline \multirow{2}{*}{ Sub Group } & \multicolumn{2}{c}{ Egger Test } & \multicolumn{2}{c}{ Begg Test } \\
\cline { 2 - 5 } & Dominant & Recessive & Dominant & Recessive \\
\hline all study & 0.14 & 0.71 & 0.47 & 0.63 \\
T1DM & 0.96 & 0.86 & 1.00 & 1.00 \\
T2DM & 0.06 & 0.62 & 0.25 & 0.46 \\
Non-Asian & 0.08 & 0.12 & 0.11 & 0.43 \\
Asian & 0.09 & 0.12 & 0.34 & 0.18 \\
\hline
\end{tabular}

\section{Discussion}

Why some diabetics develop retinopathy, whereas others do not, despite having long-term hyperglycemia, remains an undetermined question. Because known environmental factors do not fully explain this, researchers have sought the answer in the genetic background of the host [32]. The rennin-angiotensin-aldosterone system (RAAS) has been strongly implicated in the pathogenesis of progressive diabetes [64]. The RAAS is a critical regulator of sodium balance, extracellular fluid 
volume, vascular resistance, and, ultimately, arterial blood pressure by angiotensin II $[61,65,66]$. Thus, the RAAS serves as one of the most powerful regulators of arterial blood pressure and atherosclerosis and could be considered candidate genes involved in the pathogenesis of diabetic complications, including DR $[67,68]$. As the gene-encoding components of the RAAS, the ACE gene plays an important role in the RAAS, which is a complicated regulatory network with intrinsic like extrinsic agonistic and antagonistic hormones. It has been increasingly recognized that ACE inhibition demonstrates function and tissue protection of considered organs, to improve eye function of patients with diabetes mellitus and reduce the development and progression of DR [69,70]. In 1990, Rigat et al. described the polymorphism of the ACE gene based on the presence (insertion I) or absence (deletion D) of a 287 base pair element in intron 16 [11]. In plasma ACE levels, this genotype accounts for $47 \%$ of the total phenotypic variance in healthy individuals in a way that individuals with D alleles have an increased activity [11]. In addition, Danser et al. showed that the ACE I/D polymorphism also influences ACE tissue concentrations [9]. Numerous investigations into the potential role of ACE as a susceptibility gene for DR have been conducted over the past decades, with controversial results. Early meta-analyses attempted to reconcile these findings, but attempts to draw definite conclusions have been hindered by limited data, particularly when examining specific patient subgroups and increased relative studies $[56,57]$.

It is worth emphasizing that our current meta-analysis obtained several critically different conclusions from the previous reports [56,57]. In Zhou's [56] report, they conducted a separate analysis of only the T2DM and T1DM groups, which showed that the ACE genotype has a non-significant association with DR, regardless of diabetic type. Lu et al. [57] performed the meta-analysis on only the Chinese population, without any subgroup analysis on DM type and ethnicity. However, from the present meta-analysis of 40 studies reported from 1994 to 2016 and comprising 10,168 subjects, we not only found the main effects of ACE I/D polymorphism on DR risk, but also found a significant relationship in the T2DM group. From the stratification analysis by ethnicity and DM type, we found that the ACE I/D polymorphism was significantly associated with DR risk in the T2DM and Asian groups, especially in the Asian group with T2DM. These findings may indicate that genetic factors may have more impact on the Asian population with T2DM, rather than on other subgroups like the T1DM and Non-Asian population.

We conducted a comprehensive meta-analysis on 40 published studies with 4252 diabetic retinopathy cases and 5916 controls relating the variant of the ACE I/D to the risk of DR, which can provide better ability to detect smaller effect sizes. Its strength was based on the accumulation of published data, giving greater information to detect significant differences. In order to estimate the power of the study, we used the Power and Precision 4 software to conduct the power calculation by respectively accumulating the frequency of ACE D allele in case and control groups from all studies, and the result showed the power of our study is $80.2 \%$.

In this study, the effect of separate pairwise comparisons, allele contrast, and the dominant and recessive genetic models were evaluated. Substratification analysis by DM type andethnicity, and sensitivity analysis for studies not in HWE, was performed. In addition, we further evaluated the source of heterogeneity and the publication bias of included literatures.

Despite this, we still have some limits. In the meta-analysis, non-English/Chinese, non-indexed, and non-published studies literature was not reviewed, thus, some bias might be introduced [71]; only the unadjusted pooled ORs were calculated, since data for probable confounding factors that influence the estimates of associations (e.g., age, sex, BMI) were not provided; sampling variability and stratification in genetic association studies could be a possible confounding factor in the role of genetic markers. In addition, the risk effect may depend on the interaction with other risk factors: diabetes duration, $\mathrm{HbA}_{1 \mathrm{c}}$, blood pressure, total serum cholesterol, control of diabetes, and body mass index, all of which modulate the development of DR [3]. Furthermore, small numbers of individuals and inadequate information of lifestyle factors and dietary intake by the published studies limited our statistic power to fully investigate the gene-environment interactions [61]. Therefore, further 
well-designed large studies, particularly referring to GWAS and gene-environment interactions are warranted to determinate the real contribution of these polymorphisms to DR risk susceptibility and might further indicate the genetics of DR.

\section{Conclusions}

In conclusion, the present meta-analysis finds an association between DR and ACE I/D polymorphism, especially in the Asian group with T2DM. Prospective and more genome-wide association studies (GWAS) are needed to clarify the real role of the ACE gene in determining susceptibility to DR.

Acknowledgments: This work was supported by the Surface Project of the Nanjing Medical University (2012NJMU255).

Author Contributions: Zhifeng Wu and Furu Wang conceived and designed the experiments; Shasha Luo and Chao Shi performed the experiments; Furu Wang and Chao Shi analyzed the data. Furu Wang contributed reagents/materials/analysis tools; Shasha Luo and Chao Shi wrote the paper.

Conflicts of Interest: The authors declare no conflict of interest.

\section{References}

1. Cheung, N.; Mitchell, P.; Wong, T.Y. Diabetic retinopathy. Lancet 2010, 376, 124-136. [CrossRef]

2. Bourne, R.R.; Stevens, G.A.; White, R.A.; Smith, J.L.; Flaxman, S.R.; Price, H.; Jonas, J.B.; Keeffe, J.; Leasher, J.; Naidoo, K.; et al. Causes of vision loss worldwide, 1990-2010: A systematic analysis. Lancet Glob. Health 2013, 1, e339-e349. [CrossRef]

3. Yau, J.W.; Rogers, S.L.; Kawasaki, R.; Lamoureux, E.L.; Kowalski, J.W.; Bek, T.; Chen, S.J.; Dekker, J.M.; Fletcher, A.; Grauslund, J.; et al. Global prevalence and major risk factors of diabetic retinopathy. Diabetes Care 2012, 35, 556-564. [CrossRef] [PubMed]

4. Klein, R.; Klein, B.E.; Moss, S.E.; Linton, K.L. The Beaver Dam Eye Study. Retinopathy in adults with newly discovered and previously diagnosed diabetes mellitus. Ophthalmology 1992, 99, 58-62. [CrossRef]

5. Klein, R.; Klein, B.E.; Moss, S.E.; Cruickshanks, K.J. Relationship of hyperglycemia to the long-term incidence and progression of diabetic retinopathy. Arch. Intern. Med. 1994, 154, 2169-2178. [CrossRef] [PubMed]

6. Klein, R.; Klein, B.E.; Moss, S.E.; Cruickshanks, K.J. The Wisconsin epidemiologic study of diabetic retinopathy: XVII. The 14-year incidence and progression of diabetic retinopathy and associated risk factors in type 1 diabetes. Ophthalmology 1998, 105, 1801-1815. [CrossRef]

7. Nathan, D.M. Long-term complications of diabetes mellitus. N. Engl. J. Med. 1993, 328, 1676-1685. [CrossRef] [PubMed]

8. Cho, H.; Sobrin, L. Genetics of diabetic retinopathy. Curr. Diabetes Rep. 2014, 14, 515. [CrossRef] [PubMed]

9. Danser, A.H.; Schalekamp, M.A.; Bax, W.A.; van den Brink, A.M.; Saxena, P.R.; Riegger, G.A.; Schunkert, H. Angiotensin-converting enzyme in the human heart. Effect of the deletion/insertion polymorphism. Circulation 1995, 92, 1387-1388. [CrossRef] [PubMed]

10. Migdalis, I.N.; Iliopoulou, V.; Kalogeropoulou, K.; Koutoulidis, K.; Samartzis, M. Elevated serum levels of angiotensin-converting enzyme in patients with diabetic retinopathy. South. Med. J. 1990, 83, 425-427. [CrossRef] [PubMed]

11. Rigat, B.; Hubert, C.; Alhenc-Gelas, F.; Cambien, F.; Corvol, P.; Soubrier, F. An insertion/deletion polymorphism in the angiotensin I-converting enzyme gene accounting for half the variance of serum enzyme levels. J. Clin. Investig. 1990, 86, 1343-1346. [CrossRef] [PubMed]

12. Tiret, L.; Rigat, B.; Visvikis, S.; Breda, C.; Corvol, P.; Cambien, F.; Soubrier, F. Evidence, from combined segregation and linkage analysis, that a variant of the angiotensin I-converting enzyme (ACE) gene controls plasma ACE levels. Am. J. Hum. Genet. 1992, 51, 197-205. [PubMed]

13. Crisan, D.; Carr, J. Angiotensin I-converting enzyme: Genotype and disease associations. J. Mol. Diagn. 2000, 2, 105-115. [CrossRef]

14. Marre, M.; Bernadet, P.; Gallois, Y.; Savagner, F.; Guyene, T.T.; Hallab, M.; Cambien, F.; Passa, P.; Alhenc-Gelas, F. Relationships between angiotensin I converting enzyme gene polymorphism, plasma levels, and diabetic retinal and renal complications. Diabetes 1994, 43, 384-388. [CrossRef] [PubMed] 
15. Fujisawa, T.; Ikegami, H.; Shen, G.Q.; Yamato, E.; Takekawa, K.; Nakagawa, Y.; Hamada, Y.; Ueda, H.; Rakugi, H.; Higaki, J. Angiotensin I-converting enzyme gene polymorphism is associated with myocardial infarction, but not with retinopathy or nephropathy, in NIDDM. Diabetes Care 1995, 18, 983-985. [CrossRef] [PubMed]

16. Tarnow, L.; Cambien, F.; Rossing, P.; Nielsen, F.S.; Hansen, B.V.; Lecerf, L.; Poirier, O.; Danilov, S.; Parving, H.H. Lack of relationship between an insertion/deletion polymorphism in the angiotensin I-converting enzyme gene and diabetic nephropathy and proliferative retinopathy in IDDM patients. Diabetes 1995, 44, 489-494. [CrossRef] [PubMed]

17. Nagi, D.K.; Mansfield, M.W.; Stickland, M.H.; Grant, P.J. Angiotensin converting enzyme (ACE) insertion/deletion (I/D) polymorphism, and diabetic retinopathy in subjects with IDDM and NIDDM. Diabet Med. 1995, 12, 997-1001. [CrossRef] [PubMed]

18. Doi, Y.; Yoshizumi, H.; Yoshinari, M.; Iino, K.; Yamamoto, M.; Ichikawa, K.; Iwase, M.; Fujishima, M. Association between a polymorphism in the angiotensin-converting enzyme gene and microvascular complications in Japanese patients with NIDDM. Diabetologia 1996, 39, 97-102. [PubMed]

19. Yoshida, H.; Kuriyama, S.; Atsumi, Y.; Tomonari, H.; Mitarai, T.; Hamaguchi, A.; Kubo, H.; Kawaguchi, Y.; Kon, V.; Matsuoka, K.; et al. Angiotensin I converting enzyme gene polymorphism in non-insulin dependent diabetes mellitus. Kidney Int. 1996, 50, 657-664. [CrossRef] [PubMed]

20. Gutierrez, C.; Vendrell, J.; Pastor, R.; Llor, C.; Aguilar, C.; Broch, M.; Richart, C. Angiotensin I-converting enzyme and angiotensinogen gene polymorphisms in non-insulin-dependent diabetes mellitus. Lack of relationship with diabetic nephropathy and retinopathy in a Caucasian Mediterranean population. Metabolism 1997, 46, 976-980. [CrossRef]

21. Liu, Z.; Chen, C.; Guan, T.; Chen, H.; Li, L. The relationship between angiotensin converting enzyme gene polymorphism and diabetic nephropathy and diabetic mellitus. J. Kidney Dis. Dial. Kidney Transplant. 1997, 6, 407-410.

22. Hu, W.; Wang, L.; Liu, C. Association between ACE gene polymorphism and diabetic retinopathy. J. Cap. Univ. Med. Sci. 1998, 19, 41-44.

23. Hanyu, O.; Hanawa, H.; Nakagawa, O.; Tani, N.; Andou, N.; Aizawa, Y.; Shibata, A. Polymorphism of the angiotensin I-converting enzyme gene in diabetic nephropathy in type II diabetic patients with proliferative retinopathy. Ren. Fail 1998, 20, 125-133. [CrossRef] [PubMed]

24. Frost, D.; Pfohl, M.; Clemens, P.; Haring, H.U.; Beischer, W. Evaluation of the insertion/deletion ACE gene polymorphism as a risk factor for carotid artery intima-media thickening and hypertension in young type 1 diabetic patients. Diabetes Care 1998, 21, 836-840. [CrossRef] [PubMed]

25. Kimura, H.; Gejyo, F.; Suzuki, Y.; Suzuki, S.; Miyazaki, R.; Arakawa, M. Polymorphisms of angiotensin converting enzyme and plasminogen activator inhibitor-1 genes in diabetes and macroangiopathy1. Kidney Int. 1998, 54, 1659-1669. [CrossRef] [PubMed]

26. Rabensteiner, D.; Abrahamian, H.; Irsigler, K.; Hermann, K.M.; Kiener, H.P.; Mayer, G.; Kaider, A.; Prager, R. ACE gene polymorphism and proliferative retinopathy in type 1 diabetes: Results of a case-control study. Diabetes Care 1999, 22, 1530-1535. [CrossRef] [PubMed]

27. Solini, A.; Giacchetti, G.; Sfriso, A.; Fioretto, P.; Sardu, C.; Saller, A.; Tonolo, G.; Maioli, M.; Mantero, F.; Nosadini, R. Polymorphisms of angiotensin-converting enzyme and angiotensinogen genes in type 2 diabetic sibships in relation to albumin excretion rate. Am. J. Kidney Dis. 1999, 34, 1002-1009. [CrossRef]

28. Liao, L.; Lei, M.; Chen, H.; Han, X.; Fan, C. Studies on ACE gene insertion/deletion polymorphism, serum ACE activity, and diabetic retinopathy in type II diabetic patients. Hunan Yi Ke Da Xue Xue Bao 1999, 24, 33-36. [PubMed]

29. Xiang, K.; Zhen, T.; Sun, D.; Wen, Q.; Xu, J.; Li, J. The association of three genes of renin-angiotensin system with diabetic retinopathy. Chin. J. Diabetes 1999, 7, 5-8.

30. Wang Dawang, Y.Z.; Zhang, X.; Zhen, J.; Xu, Y.; Lin, X.; Yu, Z.; Shen, F.; Fen, W.; Chen, X.; Zhu, H. Angiotensin converting enzyme gene polymorphism and diabetic retinopathy in type 2 diabetic patients. Chin. J. Diabetes 1999, 7, 299-300.

31. Liu, J.; Jin, H.; Zhang, W.; Zhou, Y.; Liao, S.; Yang, L.; Huang, Q. Study on the relationship between angiotensin converting enzyme gene polymorphism and diabetic retinopathy. Chin. J. Ocul. Fundus Dis. $1999,15,37-38$. 
32. Van Ittersum, F.J.; de Man, A.M.; Thijssen, S.; de Knijff, P.; Slagboom, E.; Smulders, Y.; Tarnow, L.; Donker, A.J.; Bilo, H.J.; Stehouwer, C.D. Genetic polymorphisms of the renin-angiotensin system and complications of insulin-dependent diabetes mellitus. Nephrol. Dial. Transplant. 2000, 15, 1000-1007. [CrossRef] [PubMed]

33. Matsumoto, A.; Iwashima, Y.; Abiko, A.; Morikawa, A.; Sekiguchi, M.; Eto, M.; Makino, I. Detection of the association between a deletion polymorphism in the gene encoding angiotensin I-converting enzyme and advanced diabetic retinopathy. Diabetes Res. Clin. Pract. 2000, 50, 195-202. [CrossRef]

34. Kankova, K.; Muzik, J.; Karaskova, J.; Beranek, M.; Hajek, D.; Znojil, V.; Vlková, E.; Vácha, J. Duration of non-Insulin-dependent diabetes mellitus and the TNF-beta NcoI genotype as predictive factors in proliferative diabetic retinopathy. Ophthalmologica 2001, 215, 294-298. [CrossRef] [PubMed]

35. Yunhua, L. The Relationship between Angiotensin I Converting Enzyme Gene Polymorphism and Type 2 Diabetes or Vascular Complication of Diabetes in Guangxi Region; Guangxi Medical University: Nanning, China, 2000.

36. Yang, X.; Li, X.; Liu, J.; Chen, S.; Luo, H. Study of angiotensin converting enzyme gene polymorphism and non-insulin-dependent diabetes mellitus with microangiopathy correlation. Chin. J. Pract. Intern. Med. 2000, 20, 487-488.

37. Araz, M.; Yilmaz, N.; Gungor, K.; Okan, V.; Kepekci, Y.; Sükrü Aynacioglu, A. Angiotensin-converting enzyme gene polymorphism and microvascular complications in Turkish type 2 diabetic patients. Diabetes Res. Clin. Pract. 2001, 54, 95-104. [CrossRef]

38. Viswanathan, V.; Zhu, Y.; Bala, K.; Dunn, S.; Snehalatha, C.; Ramachandran, A.; Jayaraman, M.; Sharma, K. Association between ACE gene polymorphism and diabetic nephropathy in South Indian patients. JOP 2001, 2, 83-87. [PubMed]

39. Globocnik-Petrovic, M.; Hawlina, M.; Peterlin, B.; Petrovic, D. Insertion/deletion plasminogen activator inhibitor 1 and insertion/deletion angiotensin-converting enzyme gene polymorphisms in diabetic retinopathy in type 2 diabetes. Ophthalmologica 2003, 217, 219-224. [CrossRef] [PubMed]

40. Ha, S.K.; Park, H.C.; Park, H.S.; Kang, B.S.; Lee, T.H.; Hwang, H.J.; Kim, S.J.; Kim, D.H.; Kang, S.W.; Choi, K.H.; et al. ACE gene polymorphism and progression of diabetic nephropathy in Korean type 2 diabetic patients: Effect of ACE gene DD on the progression of diabetic nephropathy. Am. J. Kidney Dis. 2003, 41, 943-949. [CrossRef]

41. Crook, E.D.; Genous, L.; Oliver, B. Angiotensin-converting enzyme genotype in blacks with diabetic nephropathy: Effects on risk of diabetes and its complications. J. Investig. Med. 2003, 51, 360-365. [CrossRef] [PubMed]

42. Agardh, E.; Gaur, L.K.; Lernmark, Å.; Agardh, C.-D. HLA-DRB1, -DQA1, and -DQB1 subtypes or ACE gene polymorphisms do not seem to be risk markers for severe retinopathy in younger Type 1 diabetic patients. J. Diabetes Complicat. 2004, 18, 32-36. [CrossRef]

43. Xu, L.; Deng, Z.; Wu, Z.; He, R.; Tang, J.; Mu, H.; Bian, R.; Gu, H.; Wang, X.; Jiang, Y. The relationship of angiotenis converting enzyme (ACE) gene polymorphism and serum ACE levels with retinopathy in patients with type 2 diabetes mellitus. Chin. J. Diabetes 2003, 11, 344-347.

44. Wu, S.S.; Guo, Q.M.; Liu, G.L.; Zhang, J.; Zhao, C.F.; Ning, S.C.; Zhao, L.N.; Yu, F.; Yi, H.L. The relationship of angiotensin I-converting enzyme gene polymorphism with diabetic retinopathy and diabetes myocardial infarction. Zhonghua Yi Xue Yi Chuan Xue Za Zhi 2004, 21, 283-285. [PubMed]

45. Liao, L.; Lei, M.X.; Chen, H.L.; Guo, L.J.; Han, X.Y. Angiotensin converting enzyme gene polymorphism and type 2 diabetic retinopathy. Zhong Nan Da Xue Xue Bao Yi Xue Ban 2004, 29, 410-413. [PubMed]

46. Degirmenci, I.; Kebapci, N.; Basaran, A.; Efe, B.; Gunes, H.V.; Akalin, A.; Kurt, H.; Urhan, M.; Demirustu, C. Frequency of angiotensin-converting enzyme gene polymorphism in Turkish type 2 diabetic patients. Int. J. Clin. Pract. 2005, 59, 1137-1142. [CrossRef] [PubMed]

47. Chen, J.; Shen, M.; Wang, J.; Wu, S.; Xie, Y.; Zhang, Y.; Ye, X.; Gao, J.; Li, D. Correlation of gene polymorphisms of angiotensin converting enzyme and type 2 diabetic retinopathy. Clin. Med. China 2005, 21, 608-610.

48. Lee, S.J.; Choi, M.G. Association of manganese superoxide dismutase gene polymorphism (V16A) with diabetic macular edema in Korean type 2 diabetic patients. Metabolism 2006, 55, 1681-1688. [CrossRef] [PubMed]

49. Liang, S.; Bian, C.; Guan, H.; Chen, H. Detection of angiotensin converting enzyme gene insertion/deletion polymorphism and its relationship to diabetic retinopahty. Chin. Ophthalmic Res. 2006, 24, 654-656. 
50. Nikzamir, A.; Rashidi, A.; Esteghamati, A.; Nakhjavani, M.; Golmohammadi, T.; Khalilzadeh, O. The relationship between ACE gene insertion/deletion polymorphism and diabetic retinopathy in Iranian patients with type 2 diabetes. Ophthalmic Genet. 2010, 31, 108-113. [CrossRef] [PubMed]

51. Li, N.; Yang, X.F.; Gu, H.; Deng, Y.; Xu, J.; Ma, K.; Liu, N.P. Relationship of angiotensin converting enzyme gene polymorphism with diabetic retinopathy. Zhonghua Yan Ke Za Zhi 2013, 49, 52-57. [PubMed]

52. Saleem, S.; Azam, A.; Maqsood, S.I.; Muslim, I.; Bashir, S.; Fazal, N.; Riaz, M.; Ali, S.H.B.; Niazi, M.K.; Ishaq, M.; et al. Role of ACE and PAI-1 polymorphisms in the development and progression of diabetic retinopathy. PLoS ONE 2015, 10, e0144557. [CrossRef] [PubMed]

53. Settin, A.; El-Baz, R.; Ismaeel, A.; Tolba, W.; Allah, W.A. Association of ACE and MTHFR genetic polymorphisms with type 2 diabetes mellitus: Susceptibility and complications. J. Renin Angiotensin Aldosterone Syst. 2015, 16, 838-843. [CrossRef] [PubMed]

54. Narne, P.; Ponnaluri, K.C.; Siraj, M.; Ishaq, M. Association analysis of polymorphisms in genes related to oxidative stress in south indian type 2 diabetic patients with retinopathy. Ophthalmic Genet. 2016, 37, 1-8. [CrossRef] [PubMed]

55. Thomas, G.N.; Critchley, J.A.; Tomlinson, B.; Yeung, V.T.; Lam, D.; Cockram, C.S.; Chan, J.C.N. Renin-angiotensin system gene polymorphisms and retinopathy in chinese patients with type 2 diabetes. Diabetes Care 2003, 26, 1643-1644. [CrossRef] [PubMed]

56. Zhou, J.B.; Yang, J.K. Angiotensin-converting enzyme gene polymorphism is associated with proliferative diabetic retinopathy: A meta-analysis. Acta Diabetol. 2010, 47, 187-193. [CrossRef] [PubMed]

57. Lu, Y.; Ge, Y.; Hu, Q.; Shi, Y.; Xue, C.; Shi, Y.; Chen, S.; Huang, Z. Association between angiotensin-converting enzyme gene polymorphism and diabetic retinopathy in the Chinese population. J. Renin Angiotensin Aldosterone Syst. 2012, 13, 289-295. [CrossRef] [PubMed]

58. Zhou, Y.; Chen, Y.; Cao, X.; Liu, C.; Xie, Y. Association between plasma homocysteine status and hypothyroidism: A meta-analysis. Int. J. Clin. Exp. Med. 2014, 7, 4544-4553. [PubMed]

59. Zintzaras, E.; Lau, J. Synthesis of genetic association studies for pertinent gene-disease associations requires appropriate methodological and statistical approaches. J. Clin. Epidemiol. 2008, 61, 634-645. [CrossRef] [PubMed]

60. Higgins, J.P.; Thompson, S.G. Quantifying heterogeneity in a meta-analysis. Stat. Med. 2002, 21, 1539-1558. [CrossRef] [PubMed]

61. Wang, F.; Fang, Q.; Yu, N.; Zhao, D.; Zhang, Y.; Wang, J.; Wang, Q.; Zhou, X.; Cao, X.; Fan, X. Association between genetic polymorphism of the angiotensin-converting enzyme and diabetic nephropathy: A meta-analysis comprising 26,580 subjects. J. Renin Angiotensin Aldosterone Syst. 2012, 13, 161-174. [CrossRef] [PubMed]

62. Egger, M.; Davey Smith, G.; Schneider, M.; Minder, C. Bias in meta-analysis detected by a simple, graphical test. BMJ 1997, 315, 629-634. [CrossRef] [PubMed]

63. Begg, C.B.; Mazumdar, M. Operating characteristics of a rank correlation test for publication bias. Biometrics 1994, 50, 1088-1101. [CrossRef] [PubMed]

64. Schmidt, S.; Ritz, E. Genetics of the renin-angiotensin system and renal disease: A progress report. Curr. Opin. Nephrol. Hypertens. 1997, 6, 146-151. [CrossRef] [PubMed]

65. Burns, K.D. Angiotensin II and its receptors in the diabetic kidney. Am. J. Kidney Dis. 2000, 36, 449-467. [CrossRef] [PubMed]

66. Kim, S.; Iwao, H. Molecular and cellular mechanisms of angiotensin II-mediated cardiovascular and renal diseases. Pharmacol. Rev. 2000, 52, 11-34. [PubMed]

67. Ye, S.; Dhillon, S.; Seear, R.; Dunleavey, L.; Day, L.B.; Bannister, W.; Day, I.N.; Simpson, I. Epistatic interaction between variations in the angiotensin I converting enzyme and angiotensin II type 1 receptor genes in relation to extent of coronary atherosclerosis. Heart 2003, 89, 1195-1199. [CrossRef] [PubMed]

68. Tsai, C.T.; Hwang, J.J.; Ritchie, M.D.; Moore, J.H.; Chiang, F.T.; Lai, L.P.; Hsu, K.L.; Tseng, C.D.; Lin, J.L.; Tseng, Y.Z. Renin-angiotensin system gene polymorphisms and coronary artery disease in a large angiographic cohort: Detection of high order gene-gene interaction. Atherosclerosis 2007, 195, 172-180. [CrossRef] [PubMed]

69. Cordonnier, D.J.; Zaoui, P.; Halimi, S. Role of ACE inhibitors in patients with diabetes mellitus. Drugs 2001, 61, 1883-1892. [CrossRef] [PubMed] 
70. Gilbert, R.E.; Krum, H.; Wilkinson-Berka, J.; Kelly, D.J. The renin-angiotensin system and the long-term complications of diabetes: Pathophysiological and therapeutic considerations. Diabet. Med. 2003, 20, 607-621. [CrossRef] [PubMed]

71. Egger, M.; Zellweger-Zahner, T.; Schneider, M.; Junker, C.; Lengeler, C.; Antes, G. Language bias in randomised controlled trials published in English and German. Lancet 1997, 350, 326-329. [CrossRef]

(C) 2016 by the authors; licensee MDPI, Basel, Switzerland. This article is an open access article distributed under the terms and conditions of the Creative Commons Attribution (CC-BY) license (http://creativecommons.org/licenses/by/4.0/). 